\title{
Effect of Seamless Nursing for Complications on Cataract Patients
}

\author{
Junnan Lin ${ }^{1}$, Xixi Yang ${ }^{1, ~ *, ~ L i s h i ~ L u o ~}{ }^{2}$ \\ ${ }^{1}$ Department of Ophthalmology and Stomatology, The First Affiliated Hospital of Jinan University, Guangzhou, China \\ ${ }^{2}$ Ophthalmology Department, The First Affiliated Hospital of Jinan University, Guangzhou, China
}

Email address:

1075184194@qq.com (Junnan Lin),344049001@qq.com (Xixi Yang), 296354125@qq.com (Lishi Luo)

${ }^{*}$ Corresponding author

\section{To cite this article:}

Junnan Lin, Xixi Yang, Lishi Luo. Effect of Seamless Nursing for Complications on Cataract Patients. American Journal of Nursing Science. Vol. 9, No. 2, 2020, pp. 59-61. doi: 10.11648/j.ajns.20200902.13

Received: January 11, 2020; Accepted: January 27, 2020; Published: February 28, 2020

\begin{abstract}
Objective: Following to Chinese cataract patients were increased in last 3 years, we evaluated the effect of seamless nursing for complications on cataract patients. Methods: 102 patients diagnosed as cataract from June 2018 to March 2019, the patients were randomly assigned to control group and intervention group, the researchers care the patients by seamless nursing and traditional nursing. We collected the information by different questionnaires which included complications, depression, anxiety and satisfaction of patients. After that, we analyze and compare the result of different groups. Result: in complications status of patient, the intervention group was much lower than that in the control group [5 (9.8\%) vs 14 (27.5\%)]. Additionally, the improvement had not greater gap in depression and anxiety (32.25 \pm 1.02 vs $48.52 \pm 3.66,32.58 \pm 2.01$ vs $49.56 \pm 5.84)$, we use the Self-Rating Anxiety Scale (SAS) and the Self-rating depression scale (SDS) to assess depression and anxiety on the patients. Conclusion: the influence of seamless nursing was better than the effect of traditional nursing, it provide better outcome of cataract treatment for the cataract patients. The seamless nursing improve the higher satisfaction score in the result, it was good for publishing of new nursing methods. But the improvement of seamless nursing was not significant in the result.
\end{abstract}

Keywords: Cataract, Nursing, Seamless Nursing

\section{Introduction}

Following to the He's report, the number of patient with cataract is trended to increase continuously as he aging population and extended life expectancy [1]. Therefore, some public health organization the improvement which were preventable vision loss due to cataract and understanding the modifiable risk factors was important $[2,3]$. Additionally, cataract remains the leading cause of blindness in middle-income and low-income countries, so we make this study to improve the effect of cataract treatment surgery through improve the nursing methods after surgery.

Some reports attempt to investigate seamless nursing for complications on cataract patients [4-6]. Despite their report robust results, but their data was difference between both of them. For example, some reports shown that nursing intervention improvement risks of complication by improvement of treatment efficiency [7,8]. Therefore, the effect of seamless nursing for complications on cataract patients is worth studying.

The aim of this study was to evaluate effect of seamless nursing on cataract patients. The hypothesis of this study was that seamless nursing provide improvement to cataract patients during treatment. The primary outcome of this study was to compared complication rate, anxiety assessment and depression assessment, and satisfaction assessment.

\section{Methods}

\subsection{Participants Enrollment and Survey Methods}

From June 2018 to March 2019, we collected the data from 120 patients with cataract, but we excluded the data of 18 patients as they lack necessary information, so we only collected valid data from 102 patients. In this study, we use randomized controlled trial to assess the effect of seamless nursing for complications on cataract patients. The patients 
were randomly assigned to a control group $(n=51)$ and a intervention group $(\mathrm{n}=51)$. We provided the traditional nursing care to the patients of control group [9]. For the intervention group, we add seamless care on the basis of traditional nursing during study. In treatment process, we collected the complication data of patients, the complications included endothelial edema, intraocular hypertension and hyphema. In addition, the patients would complete Self-Rating Anxiety Scale (SAS) and Self-rating depression scale (SDS), they provided the anxiety information and depression information to our study $[10,11,12]$. Furthermore, The cutoff score of SAS is 50, 50-59 for mild anxiety, 60-69 for moderate anxiety, and 70 or more for severe anxiety. The lower the score shown more effective the nursing measures as the less anxiety symptoms. In SDS, those below 0.5 were not depressed, 0.5-0.59 was mild to mild depression, 0.6-0.69 was moderate to severe depression, and 0.7 or above was severe depression.

\subsection{Statistical Analysis}

Statistical analysis was performed using SPSS 24.0. We used $\mathrm{X}^{2}$ and t-test to describe the result. Results were considered statistically significant using an alpha level of 0.05 . In addition, the continuous variables accord with normal distribution were described by the means with standard deviation (SD).

\section{Result}

We collected complications associated with cataract patient, the complications included endothelial edema, intraocular hypertension and hyphema (Table 1). The number of complications in the intervention group was much lower than that in the control group [5(9.8\%) vs $14(27.5 \%)$ ]. They were significantly difference between two groups.

Table 1. Complication of cataract patient.

\begin{tabular}{lllll}
\hline Projects & Endothelial Edema & Intraocular Hypertension & Hyphema & Percent \\
\hline Intervention Group $(\mathrm{n}=51)$ & 2 & 2 & 1 & $5(9.8 \%)$ \\
Control Group $(\mathrm{n}=51)$ & 7 & 4 & 3 & $14(27.5 \%)$ \\
$\mathrm{X}^{2}$ & - & - & - & 7.16 \\
P value & - & - & - & 0.007 \\
\hline
\end{tabular}

As shown in Table 2, SAS and SDS shown that the intervention group which received seamless nursing had better outcome than the control group after the nursing in 3 days. Although the different of outcome was not significant
(32.25 \pm 1.02 vs $48.52 \pm 3.66,32.58 \pm 2.01$ vs $49.56 \pm 5.84)$, the statistical results of the outcome were statistically different in both of groups $(\mathrm{p}<0.005)$.

Table 2. The outcome of SAS and SDS.

\begin{tabular}{|c|c|c|c|c|c|c|c|c|}
\hline \multirow{2}{*}{ Projects } & \multicolumn{2}{|l|}{ SAS } & \multirow{2}{*}{$\mathbf{T}$} & \multirow{2}{*}{$P$ value } & \multicolumn{2}{|l|}{ SDS } & \multirow{2}{*}{$\mathbf{T}$} & \multirow{2}{*}{$P$ value } \\
\hline & BN & FN & & & $\mathbf{B N}$ & FN & & \\
\hline Intervention Group $(\mathrm{n}=51)$ & $62.58 \pm 5.14$ & $32.25 \pm 1.02$ & 40.9267 & $<0.005$ & $63.55 \pm 5.41$ & $32.58 \pm 2.01$ & 37.9447 & $<0.005$ \\
\hline Control Group $(\mathrm{n}=51)$ & $62.59 \pm 5.18$ & $48.52 \pm 3.66$ & 15.6861 & $<0.005$ & $63.56 \pm 5.45$ & $49.56 \pm 5.84$ & 12.3930 & $<0.005$ \\
\hline $\mathrm{T}$ & 0.010 & 30.280 & - & - & 0.0092 & 19.4402 & - & - \\
\hline$P$ value & 0.992 & $<0.005$ & - & - & 0.071 & $<0.005$ & - & - \\
\hline
\end{tabular}

SAS $=$ Self-Rating Anxiety Scale

SDS $=$ Self-rating depression scale

$\mathrm{BN}=$ before the nursing intervention

$\mathrm{FN}=$ after the nursing intervention in 3 days

We collected the satisfaction of patients by questionnaires after carrying out nursing intervention. In Table 2, the satisfaction of intervention group patients was higher slightly than the satisfaction of control group ( $92.2 \%$ vs $80.4 \%)$. Few patients provided bad assessment in satisfaction (4 out of 51 in intervention group, 10 out of 51 in control group). Compared with control group, intervention group had more very well assessment from patients.

Table 3. The satisfaction of patients.

\begin{tabular}{lllll}
\hline Projects & Very Well & Good & Bad & Percent \\
\hline Intervention Group $(\mathrm{n}=51)$ & 26 & 21 & 4 & $92.2 \%$ \\
Control Group $(\mathrm{n}=51)$ & 22 & 19 & 10 & $80.4 \%$ \\
$\mathrm{X}^{2}$ & - & - & - & 7.485 \\
P value & - & - & - & 0.006 \\
\hline
\end{tabular}

\section{Discussion}

To assess the effect of seamless nursing for cataract patients, we established a randomized controlled trial. The results indicated that seamless nursing can improve the outcome of cataract treatment in some part, both of the mental health of patient and their complications was 
improved after seamless nursing.

As shown in Table 1, the results indicated intervention group had fewer complications compared with control group. As shown in Table 2 and Table 3, better mental health in the patients with seamless nursing than that of the patients with common nursing intervention, and the patients with seamless nursing provided better outcome in satisfaction assessment compared with the patients with common nursing intervention.

Following to the result of our research, the outcomes of cataract treatment were that the patients was improved in both of groups. Although the gap between the effect of two groups was not too different, the seamless nursing had better and effective influence than traditional nursing in result of cataract treatment. Additionally, the seamless nursing make the satisfaction of cataract patients to better, although the proportion between very well level and good level was similar in both of two groups, the overall satisfaction of intervention group was better than the satisfaction of control group.

Several studies in related fields clearly demonstrated that suitable nursing intervention can improve treatment outcome of cataract patient. [13, 14, 15]. Cataracts are the most common eye disease and a major cause of visual impairment and blindness worldwide [16]. For similar, increased cataract patients were received with attention in government as increased old popular [17, 18]. Likewise, we found that the patients who received seamless nursing had better outcome in this study.

In limitation, our subject were patients with cataracts, so it is not known whether our results are application to the other diseases.

\section{Conclusion}

In conclusion, the influence of seamless nursing was better than the effect of traditional nursing, it provide better outcome of cataract treatment for the cataract patients. However, the improvement of seamless nursing was not significant in the result, it may lead to the hospital do like to use seamless nursing in nursing process. Because the seamless nursing would spend more cost than traditional nursing to get less influence. In addition, the seamless nursing improve the higher satisfaction score in the result, it was good for publishing of new nursing methods.

\section{References}

[1] He, M., Wang, W., Huang, W. Variations and trends in health burden of visual impairment due to cataract: a global analysis. Investig. Ophthalmol. Vis. Sci. 2017; 58 (10): 4299-4306.
[2] Flaxman, S. R., Bourne, R. R. A., Resnikoff, S., Ackland, P., Braithwaite, T., Cicinelli, M. V., et al. Vision Loss Expert Group of the Global Burden of Disease Study. Global causes of blindness and distance vision impairment 1990-2020: a systematic review and meta-analysis. Lancet Glob. Health 2017; 5 (12): e1221-e1234.

[3] Wu Z, Meng D, Fang C, Li J, Zheng X, Lin J, Zeng H, Lv S, Zhang Z, Luan B, Zhong Z, Chen J. PITX3 mutations associated with autosomal dominant congenital cataract in the Chinese population. Mol. Med. Rep. 2019; 19 (4): 3123-3131.

[4] Ram J, Singh R, Gupta R, Bhutani G, Gupta PC, Sukhija J. Toric intraocular lens implantation in children with developmental cataract and preexisting corneal astigmatism. Acta Ophthalmol. 2017; 95 (2): e95-e100.

[5] Guerreiro M. J. S., Erfort M. V., Henssler J., et al., Increased visual cortical thickness in sight-recovery individuals, Hum. Brain Mapp. 2016; 36 (12): 5265-5274.

[6] Yardley AM, Ali A, Najm-Tehrani N, Mireskandari K. Refractive and visual outcomes after surgery for pediatric traumatic cataract. J Cataract Refract Surg. 2018; 44 (1): 85-90.

[7] Rao DP, John PJ, Ali MH, et al. Outcomes of lensectomy and risk factors for failure in spherophakic eyes with secondary glaucoma. Br J Ophthalmol. 2018; 102 (6): 790-795.

[8] Cumberland PM, Rahi JS. Visual function, social position, and health and life chances: the UK Biobank Study. JAMA Ophthalmol. 2016; 134: 959-966.

[9] White D, Leach C, Sims R, Atkinson M, Cottrell D. Validation of the Hospital Anxiety and Depression Scale for use with adolescents. Br J Psychiatry 1999; 175: 452-454.

[10] El-Rufaie O, Absood G. Validity study of the Hospital Anxiety and Depression Scale among a group of Saudi patients. Br J Psychiatry 1987; 151: 687-688.

[11] Tan AG, Kifley A, Mitchell P, et al. Associations between methylenetetrahydrofolate reductase polymorphisms, serum homocysteine levels, and incident cortical cataract. JAMA Ophthalmol 2016; 134: 522-528.

[12] Shunmoogam N, Naidoo P, Chilton R. Paraoxonase (PON)-1: a brief overview on genetics, structure, polymorphisms and clinical relevance. Vascular Health Risk Manage 2018; 14: 137.

[13] Yu A, Lin C, Wang Q, Zheng M, Qin X. Safety of femtosecond laserassisted cataract surgery: assessment of aqueous humour and lens capsule. Acta Ophthalmol. 2016; 94 (0): 534-540.

[14] Pascolini, D., Mariotti, S. P. Global estimates of visual impairment: 2010. Br. J. Ophthalmol. 2012; 96 (5), 614-618.

[15] Shiels A, Hejtmancik JF. Mutations and mechanisms in congenital and age-related cataracts. Exp Eye Res 2017; 156: 95-102.

[16] Yang Z, Li Q, Ma X, Zhu SQ. Mutation analysis in Chinese families with autosomal dominant hereditary cataracts. Curr Eye Res 2015; 40 (12): 1225-1231. 